\title{
USO DE MÉTODOS ANTICONCEPCIONAIS POR MULHERES ADOLESCENTES DE ESCOLA PÚBLICA*
}

\author{
Larissa de Andrade Silva Ramos ${ }^{1}$, Eliel dos Santos Pereira², Kelvya Fernanda Almeida Lago \\ Lopes $^{3}$, Augusto Cezar Antunes de Araujo Filho ${ }^{4}$, Naiara Coelho Lopes ${ }^{5}$
}

\begin{abstract}
RESUMO: Objetivo: identificar o uso de métodos contraceptivos por adolescentes de uma escola pública do interior do Maranhão. Método: estudo de abordagem quantitativa, descritivo-exploratório, de corte transversal, realizadoentre os meses de novembro e dezembro de 2015, com adolescentes do ensino médio de uma escola publicado interior maranhense. Foi realizada a análise estatística descritiva dos dados. Resultados: das adolescentes, $199(88,1 \%)$ referiram possuir informações sobre métodos contraceptivos, que foram obtidas principalmente com as mães, por 139 (69,8\%). Das jovens que possuíam informações, $184(92,5 \%)$ relataram conhecer a camisinha masculina. Das adolescentes que já tinham iniciado a vida sexual, algumas referiram não ter utilizado qualquer método, apesar das informações, e 59 $(76,6 \%)$ jovens relataram ter utilizado a camisinha masculina. Conclusão: há necessidade de desenvolver constantemente ações de saúde, a fim de oportunizar que os adolescentes exerçam sua sexualidade de maneira mais segura, para evitar infecções sexualmente transmissíveis e gravidez não planejada.
\end{abstract}

DESCRITORES: Anticoncepção; Adolescente; Saúde Reprodutiva; Saúde Pública; Enfermagem.

\section{USE OF CONTRACEPTIVE METHODS BY ADOLESCENT WOMEN OF A PUBLIC SCHOOL}

ABSTRACT: Objective: to identify the use of contraceptive methods by adolescents of a public school in the state of Maranhão. Method: a quantitative, descriptive-exploratory, cross-sectional study was conducted between November and December 2015, with high school adolescents from a public school in the state of Maranhão. Descriptive statistical analysis of the data was performed. Results: of the adolescents, 199 (88.1\%) reported having information on contraceptive methods, which was mainly obtained from their mothers, by 139 (69.8\%). Of the young women who had information, $184(92.5 \%)$ reported knowing about the male condom. Of the adolescents who had already started sexual activity, some reported not using any method, despite having the information, and 59 (76.6\%) young women reported having used the male condom. Conclusion: there is a need to constantly develop health actions in order to allow adolescents to exercise their sexuality more safely, so they avoid sexually transmitted infections and unplanned pregnancies.

KEYWORDS: Contraception; Adolescent; Reproductive Health; Public Health; Nursing.

\section{USO DE MÉTODOS ANTICONCEPTIVOS EN MUJERES ADOLESCENTES DE ESCUELA PÚBLICA}

RESUMEN: Objetivo: Identificar el uso de métodos contraceptivos en adolescentes de una escuela pública del interior de Maranhão. Método: Estudio de abordaje cuantitativo, descriptivo-exploratorio, de corte transversal, realizado entre noviembre y diciembre de 2015 con adolescentes cursando enseñanza secundaria en escuela pública del interior de Maranhão. Se realizó análisis estadístico descriptivo de los datos. Resultados: Entre las adolescentes, $199(81,1 \%)$ refirieron tener información sobre métodos anticonceptivos, transmitida especialmente por sus madres en 139 (69,8\%) casos). De las jóvenes que tenían información, 184 (92,5\%) expresó que conoce el preservativo masculino. De las adolescentes que habían iniciado su vida sexual, algunas manifestaron no haber utilizado ningún método, aún contando con información, y 59 (76,6\%) jóvenes informaron haber utilizado preservativo masculino. Conclusión: Es necesario desarrollar constantemente acciones de salud buscando que los adolescentes ejerzan su sexualidad de manera más segura, para evitar infecciones sexualmente contagiosas y embarazos no planificados.

DESCRIPTORES: Anticoncepción; Adolescente; Salud Reproductiva; Salud Pública; Enfermería.

\footnotetext{
"Artigo extraído do trabalho de conclusão de Curso intitulado: Planejamento reprodutivo: o conhecimento sobre os fatores que levam a não adesão das adolescentes. Universidade Estadual do Maranhão, 2016.

${ }^{1}$ Enfermeira. Especialista em Docência do Ensino Superior. Grajaú, MA, Brasil.

${ }^{2}$ Enfermeiro. Mestre em Bioengenharia. Doutorando em Biotecnologia pela Rede Nordeste de Biotecnologia/Universidade Federal do Piauí. Docente do Departamento de Enfermagem da Universidade Estadual do Maranhão. Grajaú, MA, Brasil.

${ }^{3}$ Enfermeira. Mestre em Saúde da Família. Docente do Departamento de Enfermagem da Universidade Estadual do Maranhão. Caxias, MA, Brasil.

${ }^{4}$ Enfermeiro. Mestre em Enfermagem. Discente do curso de Doutorado do Programa de Pós-Graduação em Enfermagem da Universidade Federal do Piauí. Teresina, PI, Brasil.

${ }^{5}$ Enfermeira. Especialista em Gestão e Auditoria em Serviços de Saúde. Grajaú, MA, Brasil. 


\section{INTRODUÇÃO}

A Organização Mundial de Saúde compreende a adolescência como o período entre 10 e 19 anos de idade, no qual acontecem várias mudanças de ordem biológica, psicológica ou social(1), as quais produzem incertezas, inseguranças, dúvidas, descobertas sobre si e sobre a própria sexualidade ${ }^{(2-3)}$.

Tais mudanças encontram-se relacionadas ao crescimento físico, à maturação sexual e à capacidade de reprodução ${ }^{(2,4)}$ e, por isso, nesta fase da vida tende a ocorrer o início da vida sexual para uma importante parcela deste grupo ${ }^{(5)}$, que, conforme a Pesquisa Nacional de Saúde do Escolar (PeNSE, 2016), geralmente acontece entre os 13 e 15 anos, especialmente no sexo masculino ${ }^{(6)}$.

Sabe-se que a sexualidade encontra-se intrínseca na adolescência, e é algo que vai além do biológico, sendo considerada, assim, um fenômeno psicológico e social, tendo em vista que é influenciada por fatores socioculturais, como as crenças e os valores ${ }^{(3)}$, os quais desempenham funções preditoras no comportamento sexual dos adolescentes ${ }^{(4)}$. Assim, a sexualidade na adolescência torna-se um tema importante para a saúde pública, tendo em vista que esse período é marcado por comportamentos e atitudes capazes de potencializar as vulnerabilidades ${ }^{(4,7)}$.

Neste sentido, entende-se que o exercício da sexualidade na adolescência pode acarretar consequências negativas à vida do adolescente, que interfiram no seu modo de vida, como gravidez precoce e indesejada, Infecções Sexualmente Transmissíveis (IST), e, também, limitar o desempenho escolar desses jovens ${ }^{(2-4,8)}$. Deste modo,considera-se necessário o incentivo, antes do início da vida sexual, do uso de métodos contraceptivos, e, também, de uma orientação que compreenda o adolescente como coparticipe do seu cuidado, a fim de atuar na prevenção da gravidez precoce e indesejada, e das $\mathrm{IST}^{(2-3,8)}$.

Assim, tendo em vista a relevância da temática e a importância do uso dos métodos contraceptivos como um aliado para a prevenção de fatores que interfiram no modo de vida dos jovens, este trabalho teve como objetivo identificar o uso de métodos contraceptivos por adolescentes de uma escola pública do interior do Maranhão.

\section{METODOLOGIA}

Trata-se de um estudo de abordagem quantitativa, descritivo-exploratório, de corte transversal, realizado no período de 30 de novembro a 4 de dezembro de 2015, em uma escola pública do interior do estado do Maranhão, quefuncionava, no período da coleta, com 696 alunos distribuídos nos três turnos.

Os critérios de inclusão foram: adolescentes do sexo feminino, que estivessem regularmente matriculadas na instituição pública e que cursassem o Ensino Médio, no ano de 2015. Assim, de um total de 299 adolescentes do sexo feminino, ressalta-se que a população foi constituída por 226 delas, pois foram excluídas 16 por não estarem presentes em sala de aula no momento da coleta e 57 por apresentarem idade superior a 19 anos.

A coleta dos dados aconteceu em cada sala de aula, realizada com um questionário estruturado e autoaplicável dividido em duas etapas. A primeira permite a caracterização dos sujeitos, com variáveis como idade, cor, estado civil, religião, escolaridade, moradia, filhos e a renda familiar. A segunda tratava sobre o planejamento reprodutivo das adolescentes, envolvendo o conhecimento, a utilização e acessibilidade aos métodos contraceptivos.

Os dados, após a coleta, foram processados e analisados utilizando-se o programaMicrosoft ExcelOO, no qual se realizou a análise estatística descritiva.Destaca-se que nas variáveis 'fontes de informação', 'métodos conhecidos', 'métodos utilizados' e 'local de acesso' as adolescentes podiam elencar mais de uma alternativa, configurando-se, portanto, em uma variável de resposta múltipla. 
Ressalta-se que este estudo teve autorização do Comitê de Ética em Pesquisa (CEP) da Universidade Estadual do Maranhão, parecer $\mathrm{n} . \stackrel{0}{1.286 .760}$, firmando o compromisso com o respeito pela dignidade humana.

\section{RESULTADOS}

Com relação às características sociodemográficas, observou-se que $58(25,7 \%)$ adolescentes tinham 16 anos de idade, 171 (75,7\%) se autodeclararam de cor parda, $181(80,1 \%)$ eram solteiras, 133 (58,8\%) não tinham iniciado a vida sexual, $211(93,4 \%)$ não possuíam filhos, 77 (34,1\%) estavam cursando a $2^{\text {a }}$ série do ensino médio, 143 (63,3\%) eram católicas,149 (65,9\%) adolescentes residiam com os pais e $111(49,1 \%)$ tinhamrenda de um salário mínimo (Tabela 1).

Tabela 1 - Características sociodemográficas de adolescentes matriculadas em uma escola pública do

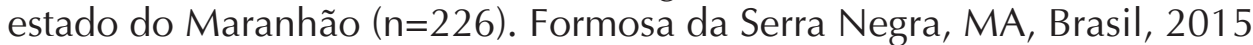

\begin{tabular}{|c|c|c|}
\hline Variável & $\mathbf{n}$ & $\%$ \\
\hline \multicolumn{3}{|l|}{ Idade } \\
\hline 14 & 12 & 5,3 \\
\hline 15 & 48 & 21,2 \\
\hline 16 & 58 & 25,7 \\
\hline 17 & 56 & 24,8 \\
\hline 18 & 32 & 14,2 \\
\hline 19 & 20 & 8,8 \\
\hline \multicolumn{3}{|l|}{ Cor } \\
\hline Branca & 50 & 22,1 \\
\hline Parda & 171 & 75,7 \\
\hline Negra & 5 & 2,2 \\
\hline \multicolumn{3}{|l|}{ Estado civil } \\
\hline Solteira & 181 & 80,1 \\
\hline Casada & 29 & 12,8 \\
\hline União Estável & 16 & 7,1 \\
\hline \multicolumn{3}{|l|}{ Religião } \\
\hline Católica & 143 & 63,3 \\
\hline Protestante & 77 & 34,1 \\
\hline Outros & 6 & 2,6 \\
\hline \multicolumn{3}{|l|}{ Série do ensino médio } \\
\hline $1^{\text {a }}$ Série & 75 & 33,2 \\
\hline $2^{a}$ Série & 77 & 34,1 \\
\hline $3^{\text {a }}$ Série & 74 & 32,7 \\
\hline \multicolumn{3}{|l|}{ Moram com os pais } \\
\hline Sim & 149 & 65,9 \\
\hline Não & 77 & 34,1 \\
\hline \multicolumn{3}{|l|}{ Iniciou vida sexual } \\
\hline Sim & 93 & 41,2 \\
\hline Não & 133 & 58,8 \\
\hline \multicolumn{3}{|l|}{ Possui filhos } \\
\hline Sim & 15 & 6,6 \\
\hline Não & 211 & 93,4 \\
\hline \multicolumn{3}{|l|}{ Renda familiar } \\
\hline < Salário Mínimo & 72 & 31,9 \\
\hline = Salário Mínimo & 111 & 49,1 \\
\hline > Salário Mínimo & 43 & 19 \\
\hline
\end{tabular}


Verificou-seo predomínio da existência de informação, em todas as idades, sobre algum método contraceptivo,199 (88,1\%).Com relação ao uso dos métodos, 149(65,9\%), relataram não terem utilizado nenhum métodocontraceptivo até o momentoda coleta de dados (Tabela 2).

Tabela 2 - Informação e utilizaçãodealgum método contraceptivo por adolescentes matriculadas em escola pública do estado do Maranhão, por idade (n=226). Formosa da Serra Negra, MA, Brasil, 2015

\begin{tabular}{|c|c|c|c|c|c|c|c|c|c|c|c|c|c|c|}
\hline \multirow{3}{*}{ Variável } & \multicolumn{12}{|c|}{ Idade } & & \\
\hline & \multicolumn{2}{|c|}{14} & \multicolumn{2}{|c|}{15} & \multicolumn{2}{|c|}{16} & \multicolumn{2}{|c|}{17} & \multicolumn{2}{|c|}{18} & \multicolumn{2}{|c|}{19} & \multicolumn{2}{|c|}{ Total } \\
\hline & $\mathbf{N}$ & $\%$ & $\mathbf{n}$ & $\%$ & $\mathbf{n}$ & $\%$ & $\mathbf{n}$ & $\%$ & $\mathbf{n}$ & $\%$ & $\mathbf{n}$ & $\%$ & $\mathbf{n}$ & $\%$ \\
\hline \multicolumn{15}{|c|}{ Possui informação } \\
\hline Sim & 11 & 4,9 & 39 & 17,3 & 50 & 22,1 & 51 & 22,6 & 30 & 13,3 & 18 & 8 & 199 & 88,1 \\
\hline Não & 1 & 0,4 & 9 & 4 & 8 & 3,5 & 5 & 2,2 & 2 & 0,9 & 2 & 0,9 & 27 & 11,9 \\
\hline \multicolumn{15}{|c|}{ Usou algum método } \\
\hline Sim & 2 & 0,9 & 7 & 3,1 & 20 & 8,8 & 23 & 10,2 & 13 & 5,8 & 12 & 5,3 & 77 & 34,1 \\
\hline Não & 10 & 4,4 & 41 & 18,1 & 38 & 16,8 & 33 & 14,6 & 19 & 8,4 & 8 & 3,5 & 149 & 65,9 \\
\hline
\end{tabular}

Observou-se que das 199 adolescentes que referiram ter informação sobre métodos contraceptivos,139adolescentes (69,8\%)receberamessas informações, principalmente, das mães (Tabela $3)$.

Tabela 3 - Fontes de informação sobre métodos contraceptivos de adolescentes matriculadas em escola pública do estado do Maranhão, por idade (n=199). Formosa da Serra Negra, MA, Brasil, 2015

\begin{tabular}{|c|c|c|c|c|c|c|c|c|c|c|c|c|c|c|}
\hline \multirow{3}{*}{ Variável } & \multicolumn{12}{|c|}{ Idade } & & \\
\hline & \multicolumn{2}{|c|}{14} & \multicolumn{2}{|c|}{15} & \multicolumn{2}{|c|}{16} & \multicolumn{2}{|c|}{17} & \multicolumn{2}{|c|}{18} & \multicolumn{2}{|c|}{19} & \multicolumn{2}{|c|}{ Total } \\
\hline & $\mathbf{n}$ & $\%$ & $\mathbf{n}$ & $\%$ & $\mathbf{n}$ & $\%$ & $\mathbf{n}$ & $\%$ & $\mathbf{n}$ & $\%$ & $\mathbf{n}$ & $\%$ & $\mathbf{n}$ & $\%$ \\
\hline \multicolumn{15}{|l|}{ Fontes de informação* } \\
\hline Pai & 3 & 1,5 & 13 & 6,5 & 18 & 9 & 18 & 9 & 10 & 5 & 7 & 3,5 & 69 & 34,7 \\
\hline Mãe & 7 & 3,5 & 35 & 17,6 & 35 & 17,6 & 32 & 16,1 & 20 & 10,1 & 10 & 5 & 139 & 69,8 \\
\hline Profissional de saúde & 1 & 0,5 & 12 & 6 & 11 & 5,5 & 14 & 7 & 5 & 2,5 & 8 & 4 & 51 & 25,6 \\
\hline Tios & 2 & 1 & 10 & 5 & 15 & 7,5 & 9 & 4,5 & 5 & 2,5 & 3 & 1,5 & 44 & 22,1 \\
\hline Professores & 1 & 0,5 & 16 & 8 & 27 & 13,6 & 22 & 11,1 & 9 & 4,5 & 11 & 5,5 & 86 & 43,2 \\
\hline Amigos & 2 & 1 & 21 & 10,6 & 29 & 14,6 & 20 & 10,1 & 9 & 4,5 & 5 & 2,5 & 86 & 43,2 \\
\hline Irmãos & 1 & 0,5 & 6 & 3 & 13 & 6,5 & 6 & 3 & 3 & 1,5 & 2 & 1 & 31 & 15,6 \\
\hline Outros & - & - & 1 & 0,5 & 2 & 1,0 & - & - & 1 & 0,5 & 1 & 0,5 & 5 & 2,5 \\
\hline
\end{tabular}

*Resposta múltipla

Quanto aos métodos contraceptivos conhecidos, verificou-seque das 199 adolescentes que referiram ter informações acerca de métodos contraceptivos, $184(92,5 \%)$ adolescentes referiram conhecer a camisinha masculina, sendo este o método contraceptivo mais conhecido (Tabela 4). 
Tabela 4 - Métodos contraceptivos conhecidos por adolescentes matriculadas em escola pública do estado do Maranhão, por idade ( $n=199)$. Formosa da Serra Negra, MA, Brasil, 2015

\begin{tabular}{|c|c|c|c|c|c|c|c|c|c|c|c|c|c|c|}
\hline \multirow{3}{*}{ Variável } & \multicolumn{12}{|c|}{ Idade } & & \\
\hline & \multicolumn{2}{|c|}{14} & \multicolumn{2}{|c|}{15} & \multicolumn{2}{|c|}{16} & \multicolumn{2}{|c|}{17} & \multicolumn{2}{|c|}{18} & \multicolumn{2}{|c|}{19} & \multicolumn{2}{|c|}{ Total } \\
\hline & $\mathbf{n}$ & $\%$ & $\mathbf{n}$ & $\%$ & $\mathbf{n}$ & $\%$ & $\mathbf{n}$ & $\%$ & $\mathbf{n}$ & $\%$ & $\mathbf{n}$ & $\%$ & $\mathbf{n}$ & $\%$ \\
\hline \multicolumn{15}{|l|}{ Métodos conhecidos* } \\
\hline Camisinha masculina & 10 & 5 & 38 & 19,1 & 45 & 22,6 & 50 & 25,1 & 23 & 11,6 & 18 & 9 & 184 & 92,5 \\
\hline Camisinha feminina & 8 & 4 & 28 & 14,1 & 43 & 21,6 & 32 & 16,1 & 18 & 9 & 12 & 6 & 141 & 70,9 \\
\hline Tabelinha & 3 & 1,5 & 14 & 7 & 18 & 9 & 11 & 5,5 & 7 & 3,5 & 7 & 3,5 & 60 & 30,2 \\
\hline Pílula contraceptiva & 11 & 5,5 & 30 & 15,1 & 47 & 23,6 & 28 & 14,1 & 21 & 10,6 & 13 & 6,5 & 150 & 75,4 \\
\hline Injeção hormonal & 3 & 1,5 & 11 & 5,5 & 29 & 14,6 & 17 & 8,5 & 7 & 3,5 & 6 & 3 & 73 & 36,7 \\
\hline Pílula do dia seguinte & 9 & 4,5 & 32 & 16,1 & 41 & 20,6 & 35 & 17,6 & 15 & 7,5 & 17 & 8,5 & 149 & 74,9 \\
\hline DIU & 4 & 2 & 10 & 5 & 21 & 10,6 & 11 & 5,5 & 4 & 2 & 2 & 1 & 52 & 26,1 \\
\hline Diafragma & 2 & 1 & 6 & 3 & 9 & 4,5 & 7 & 3,5 & - & - & 2 & 1 & 26 & 13,1 \\
\hline
\end{tabular}

*Resposta múltipla

Quanto aos métodos contraceptivos utilizados pelas 77 adolescentes que já tinham iniciado a vida sexual, observou-se que a camisinha masculina foi o método mais utilizado pelasjovens,59(76,6\%), e que seu uso abrange adolescentesde todas as idades. No que diz respeito ao local de acesso, pelas adolescentes que já fizeram uso, observou-se que a escola se destacou como o principal local de acesso, $25(32,5 \%)$ (Tabela 5).

Tabela 5 - Local de acesso e métodos contraceptivosutilizados por adolescentes matriculadas em escola pública do estado do Maranhão, por idade (n=77). Formosa da Serra Negra, MA, Brasil, 2015

\begin{tabular}{|c|c|c|c|c|c|c|c|c|c|c|c|c|c|c|}
\hline \multirow{3}{*}{ Variável } & \multicolumn{12}{|c|}{ Idade } & & \\
\hline & \multicolumn{2}{|c|}{14} & \multicolumn{2}{|c|}{15} & \multicolumn{2}{|c|}{16} & \multicolumn{2}{|c|}{17} & \multicolumn{2}{|c|}{18} & \multicolumn{2}{|c|}{19} & \multicolumn{2}{|c|}{ Total } \\
\hline & $\mathbf{n}$ & $\%$ & $\mathbf{n}$ & $\%$ & $\mathbf{n}$ & $\%$ & $\mathbf{n}$ & $\%$ & $\mathbf{n}$ & $\%$ & $\mathbf{n}$ & $\%$ & $\mathbf{n}$ & $\%$ \\
\hline \multicolumn{15}{|l|}{ Métodos utilizados* } \\
\hline Camisinha masculina & 2 & 2,6 & 2 & 2,6 & 13 & 16,9 & 22 & 28,6 & 11 & 14,3 & 9 & 11,7 & 59 & 76,6 \\
\hline Camisinha feminina & - & - & - & - & 2 & 2,6 & 1 & 1,3 & 1 & 1,3 & 1 & 1,3 & 5 & 6,5 \\
\hline Tabelinha & - & - & 1 & 1,3 & 2 & 2,6 & - & - & - & - & 3 & 3,9 & 6 & 7,8 \\
\hline Pílula contraceptiva & - & - & 1 & 1,3 & 6 & 7,8 & 13 & 16,9 & 5 & 6,5 & 4 & 5,2 & 19 & 24,7 \\
\hline Injeção hormonal & - & - & 1 & 1,3 & 2 & 2,6 & 3 & 3,9 & - & - & 6 & 7,8 & 12 & 15,6 \\
\hline Pílula do dia seguinte & - & - & 5 & 6,5 & 9 & 11,7 & 18 & 23,4 & 9 & 11,7 & 12 & 15,6 & 53 & 68,8 \\
\hline \multicolumn{15}{|l|}{ Local de acesso* } \\
\hline Farmácia & - & - & 2 & 2,6 & 3 & 3,9 & 4 & 5,2 & 7 & 9,1 & 3 & 3,9 & 19 & 24,7 \\
\hline Hospital & - & - & - & - & 4 & 5,2 & 6 & 7,8 & 6 & 7,8 & 5 & 6,5 & 21 & 27,3 \\
\hline Escola & 1 & 1,3 & 5 & 6,5 & 9 & 11,7 & 5 & 6,5 & 4 & 5,2 & 1 & 1,3 & 25 & 32,5 \\
\hline Unidade Básica de Saúde & - & - & 4 & 5,2 & 4 & 5,2 & 6 & 7,8 & 5 & 6,5 & 1 & 1,3 & 20 & 26 \\
\hline Não possui acesso & 1 & 1,3 & - & - & 5 & 6,5 & 5 & 6,5 & 4 & 5,2 & 3 & 3,9 & 18 & 23,4 \\
\hline Outros & 1 & 1,3 & - & - & 1 & 5 & 1 & 1,3 & 1 & 7,7 & - & - & 4 & 5,2 \\
\hline
\end{tabular}

*resposta múltipla 


\section{DISCUSSÃO}

Neste estudo, das 226adolescentes, 199 referiram ter informações sobre algum método contraceptivo. Esse dado constitui um fator relevante, pois sabe-se que o conhecimento sobre esses métodos reduz a gravidez não planejada e as $\mathrm{IST}^{(9)}$. Entretanto, observou-se que uma parcela significativa ainda não possui informações. Por isso, a fim de que todos os adolescentes possuam condições de desfrutar de sua sexualidade de maneira saudável, com responsabilidade e segurança, considera-se imperativo que os profissionais de saúde promovam cotidianamente ações que abordem a saúde sexual e reprodutiva na adolescência ${ }^{(3-4)}$.

Das 226 adolescentes deste estudo,133 referiramnão ter iniciado a vida sexual. Tal fato também pode ser considerado um ponto positivo para a saúde dessas adolescentes, tendo em vista que o início precoce da vida sexualpode ocasionar repercussões negativas na saúde sexual e reprodutiva dosadolescentes ${ }^{(9-10)}$. Isso encontra-se relacionado ao fato que, muitas vezes, pela falta de conhecimento, orientação, reflexão e consciência crítica frente ao sexo, o adolescente adota um comportamento sexual sem os cuidados preventivos adequados relacionados à gravidez precoce e às $\mathrm{IST}^{(2,8-9)}$.

Quanto ao uso de algum método contraceptivo, das 93 adolescentes que haviam iniciado a vida sexual, apenas 77relataram ter utilizado. Isso evidencia que algumas adolescentes não fizeram uso de métodos contraceptivos em suas primeiras relações sexuais. Sabe-se que o não uso ou o uso não frequente de contraceptivos está associado a maiores riscos de exposição à IST e à ocorrência de uma gestação não planejada e precoce ${ }^{(11-12)}$. Esse achado, portanto, demonstra a vulnerabilidade que essa população se encontra exposta e reforça a necessidade e importância de abordagens constantesacerca dos métodos contraceptivos, sobretudo, antes da iniciação sexual ${ }^{(9,13)}$.

Logo, evidencia-se a necessidade de elaborar e efetivar ações de saúde que abordem questões referentes às vulnerabilidades e sexualidade no âmbito escolar ${ }^{(4)}$, pois um estudo com dados da PeNSE do ano de 2012 verificou que não receber informações sobre saúde sexual e reprodutiva na escola aumentou a chance de ter relação sexual, com maior magnitude para o sexo desprotegido ${ }^{(14)}$. Entendese, portanto, que é fundamental não apenas informar, mas conhecer o que os adolescentes pensam e as deficiências existentes entre o conhecimento e o uso $^{(2-3)}$.

Neste estudo, as principais fontes de informação,sobre métodos contraceptivos, referidas pelas adolescentes são as mães, seguidas dos professores e amigos. Esse achado também foi encontrado em estudos realizados em uma escola estadual de Santa Catarina ${ }^{(15)} \mathrm{e}$ em uma escola pública do Rio Grande do Sul(16), porém, difere de estudo realizado em Caxias-MA ${ }^{(17)}$, no qual se verificou como principais fontes de informação a escola, os meios de comunicação e as mães, respectivamente.

Diante desse achado, destaca-se que os jovens não têm buscado os profissionais de saúde para receberem informações sobre métodos contraceptivos, o que constitui um ponto negativo para a saúde das adolescentes, tendo em vista que um estudo, realizado em Gana ${ }^{(18)}$, verificou que aatuação profissional tem a capacidade de melhorar o uso de contraceptivos entre adolescentes do sexo feminino e nas mulheres em geral, uma vez que adolescentes que visitaram serviços de saúde ou que receberam visitas de profissionais de saúde eram mais propensas a usar qualquer contraceptivo ${ }^{(18)}$.

Destarte, considera-se que os profissionais de saúde possuem um papel fundamental nesse processo, e, por isso, devem estar promovendo constantemente ações de saúde que gerem troca de informações sobre saúde sexual e reprodutiva, a fim de empoderar as adolescentes quanto às boas práticas contraceptivas ${ }^{(9)}$. O enfermeiro, por sua vez, deve implementar medidas preventivas e efetivar ações de saúde que ajudem a reduzir a vulnerabilidade dos adolescentes quanto às IST e à gravidez precoce ${ }^{(7)}$. Para isso, ressalta-se a importância de trabalhar na perspectiva de que a sexualidade é algo intrínseco ao ser humano e que deve-se instruir os adolescentes a vivenciarem sua sexualidade de maneira mais saudável ${ }^{(11)}$.

Os métodoscontraceptivos mais conhecidos pelas adolescentes deste estudo foram a camisinha masculina e a pílula anticoncepcional, respectivamente, corroborando com estudos realizadosem algumas escolas públicas de Santa Catarina ${ }^{(15)}$ e de Bom Jesus- $\mathrm{Pl}^{(19)}$,em Unidades de Saúde da Família de Ribeirão Preto-SP(20) e em um serviço de planejamento reprodutivo de Fortaleza-CE ${ }^{(9)}$. Ésabido que o conhecimento acerca dos diferentes métodos contraceptivos pelos adolescentes vem aumentando, assim 
como é consenso que os métodos mais conhecidos pelos jovens brasileirossão,fundamentalmente,a camisinha masculina e a pílula contraceptiva ${ }^{(5,19)}$.

Dentre os métodos contraceptivos mais utilizados, o preservativo masculino e a pílula do dia seguinte ganharam destaque. Em pesquisa realizada na Colômbia, o preservativo foi referido como o métodomais utilizado pelos adolescentes ${ }^{(21)}$. Uma investigação, realizada em Porto Alegre, contrapõem os dados deste estudo, tendo em vista que os mais utilizados foram a pílula anticoncepcional e o preservativo, respectivamente ${ }^{(22)}$.

Neste estudo, a escola foi citada como o principal local de acesso aos métodos contraceptivos, dado divergente ao de estudos realizados em outros contextos, como em um realizado em Goiânia-GO(4), em que a obtenção de métodos contraceptivos apontadas pelas adolescentes foi a farmácia, a unidade de saúde e/ou parceiro ${ }^{(4)}$; e em um estudo realizado na Colômbia ${ }^{(21)}$, em que a maioria dos investigados informa que obtém os métodos na farmácia ${ }^{(21)}$.

As limitações deste estudo encontram-se relacionadas ao seu delineamento, tendo em vista que possíveis vieses podem ocorrer em estudos transversais, como vieses de informação, por falha da memória, bem como por respostas que não condizem com a realidade devido ao receio de que as informações referidas pudessem ser acessadas pelos pais ou pelas autoridades da escola, embora essa possibilidade tenha sido descartada e frisadadurante e em todas as entrevistas.

\section{CONCLUSÃO}

Os resultados deste estudo demonstram que as adolescentes possuem informações sobre os métodos contraceptivos, principalmente, a camisinha masculina e a pílula anticoncepcional. Entretanto, algumas adolescentes ainda afirmaram não possuir informações acerca desses métodos.

Torna-se oportuno destacar que, apesar da maioria possuir informações, o uso dos métodos não superou sequer o número de adolescentes que afirmou já ter iniciado a vida sexual.Ademais, observouse a visibilidade reduzida dos profissionais de saúde, os quais foram pouco citados como fontes de informação.

Essas questões apoiam a necessidade de desenvolver constantemente ações de saúde que melhorem o conhecimento dos adolescentes sobre a contracepção, a fim de que possam exercer sua sexualidade de forma mais segura. Além disso, vislumbra o envolvimento dos pais, professores e profissionais de saúde nesse processo, com o intuito de minimizar as vulnerabilidades que os adolescentes podem se expor neste período.

Destaca-se, como implicações para a enfermagem, a importância do papel do enfermeiro, sobretudo o de educador, como uma maneira de intervir nas dificuldades e fragilidades que os adolescentes possuem, a fim de que possam desempenhar plenamente a sua sexualidade de forma saudável.

\section{- REFERÊNCIAS}

1. World Health Organization(WHO). Helth Topics. Adolescent health [Internet]. WHO; 2015 [acessoem 30 jan 2018 ]. Disponível: http://who.int/topics/adolescent_health/en/.

2. de Araújo AKL, de Araujo Filho ACA, Araújo TME, Nery IS, da Rocha SS. Contracepção na adolescência: conhecimento, métodos escolhidos e critérios adotados. Rev. Pesqui. Cuid. Fundam. [Internet] 2015;7(3) [acesso em 02 jun 2017]. Disponível:http://dx.doi.org/10.9789/2175-5361.2015.v7i3.2815-2825.

3. Molina MCC, Stoppiglia PGS, Martins CBG, Alencastro LCS. Conhecimento de adolescentes do ensino médio quanto aos métodos contraceptivos. O Mundo da Saúde. [Internet] 2015;39(1) [acesso em 02 jun 2017]. Disponível:http:// dx.doi.org/10.15343/0104-7809.201539012231. 
4. Oliveira PC, Pires LM, Junqueira ALN, Vieira MAS, Matos MA, Amorim KAC et al. Conhecimento em saúde sexual e reprodutiva: estudo transversal com adolescentes. Rev. Eletr. Enf. [Internet]. 2017;19 [acesso em 30 jan 2018]. Disponível: http://dx.doi.org/10.5216/ree.v19.39926.

5. Borges ALV, Fujimori E, Kuschnir MCC, Chofakian CBN, de Moraes AJP, Azevedo GD.et al. ERICA: início da vida sexual e contracepção em adolescentes brasileiros. RevSaude Publica.[Internet] 2016;50(Suppl 1) [acesso em 05 jun 2017]. Disponível: http://dx.doi.org/10.1590/S01518-8787.2016050006686.

6. Instituto Brasileiro de Geografia e Estatística - IBGE. Pesquisa Nacional de Saúde do Escolar. Rio de Janeiro; 2016.

7. Nunes BKG, Guerra ADL, Silva SM, Guimarães RA, de Souza MM, Teles AS et al. O uso de preservativos: a realidade de adolescentes e adultos jovens de um assentamento urbano. Rev. Eletr. Enf. [Internet]. 2017;19 [acesso em 30 jan 2018]. Disponível: http://dx.doi.org/10.5216/ree.v19.39041.

8. Costa GPO, Guerra AQS, de Araújo ACPF. Conhecimentos, atitudes e práticas sobre contracepção para adolescentes. Rev. Pesqui. Cuid. Fundam. [Internet] 2016;8(1)[acesso em 06 jun 2017]. Disponível:http://dx.doi.org/10.9789/21755361.2016.v8i1.3597-3608.

9. Queiroz MVO, Vasconcelos MM, de Alcântara CM, Fé MCM, Silva ANS. Características sociodemográficas e gineco-obstétricas de adolescentes assistidas em serviço de planejamento familiar. RevEnferm UFSM. [Internet] 2017;7(4) [acesso em 30 jan 2018]. Disponível: http://dx.doi.org/10.5902/2179769226988.

10. Gonçalves H, Machado EC, Soares ALG, Camargo-Figuera FA, Seerig LM, Mesenburg MA et al. Início da vida sexual entre adolescentes (10 a 14 anos) e comportamentos em saúde. Rev. bras. epidemiol. [Internet]. 2015;18(1) [acesso 01 fev 2018]. Disponível em: http://dx.doi.org/10.1590/1980-5497201500010003.

11. Patias ND, Dias ACG. Sexarca, informação e uso de métodos contraceptivos: comparação entre adolescentes. Psico -USF. [Internet] 2014;19(1) [acesso em 18 jun 2017]. Disponível: http://www.redalyc.org/pdf/4010/401041441003.pdf

12. de Medeiros TFR, dos Santos SMP, Xavier AG, Gonçalves RL, Mariz SR, de Sousa FLP. Vivência de mulheres sobre contracepção na perspectiva de gênero. Rev Gaúcha Enferm. [Internet] 2016;37(2) [acesso em 08 jul 2017]. Disponível: http://dx.doi.org/10.1590/1983-1447.2016.02.57350.

13. Yidana A, Ziblim SD, Azongo TB, Abass YI. Socio-Cultural Determinants of Contraceptives Use Among Adolescents in Northern Ghana. Public Health Research. [Internet] 2015;5(4) [acesso em 01 fev 2018]. Disponível em: http://article. sapub.org/10.5923.j.phr.20150504.01.html.

14. Oliveira-Campos M, Nunes ML, Madeira FC, Santos MG, Bregmann SR, Malta DC et al. Comportamento sexual em adolescentes brasileiros, Pesquisa Nacional de Saúde do Escolar (PeNSE 2012). Rev. bras. epidemiol. [Internet] 2014;17(Suppl 1) [acesso em 30 jan 2018]. Disponível em: http://dx.doi.org/10.1590/1809-4503201400050010.

15. Madureira VSF, Weber AI. Conhecimento de adolescentes mulheres sobre contracepção. CogitareEnferm. [Internet] 2011;16(2) [acesso em 25 jul 2017]. Disponível: http://dx.doi.org/10.5380/ce.v16i2.20234.

16. Genz N, Meincke SMK, Carret MLV, Corrêa ACL, Alves CN. Doenças sexualmente transmissíveis: conhecimento e comportamento sexual de adolescentes. Texto contexto - enferm. [Internet] 2017; 26(2) [acesso em 02 fev 2018 ]. Disponível em: http://dx.doi.org/10.1590/0104-07072017005100015.

17. Portela NLC, Albuquerque LPA. Adolescência: fontes de informações sobre métodos contraceptivos.RevEnferm UFPI. [Internet] 2014;3(1) [acesso em 07 ago 2017]. Disponível: http://www.ojs.ufpi.br/index.php/reufpi/article/ view/1362/pdf.

18. Nyarko SH. Prevalence and correlates of contraceptive use among female adolescents in Ghana. BMC Women's Health. [Internet] 2015; 15(60) [acessoem 31 jan 2018]. Disponível: http://dx.doi.org/10.1186/s12905-015-0221-2

19. Oliveira KNS, Oliveira KNS, Bezerra MAR, Rocha RC, Santos LR, Saraiva PVS. Educação sexual na adolescência e juventude: abordando as implicações da sexualidade no contexto escolar. Sanare. [Internet] 2013;12(2) [acesso em 31 jan 2018]. Disponível: https://sanare.emnuvens.com.br/sanare/article/view/376/268. 
20. Zanini M, Selvante JDS, Quagliato FF. Uso de contraceptivos e fatores associados entre adolescentes de 15 a 18 anos de idade em Unidade de Saúde da Família. RevMed (São Paulo). [Internet] 2017;96(1) [acesso em 31 jan 2018 ]. Disponível: http://dx.doi.org/10.11606/issn.1679-9836.v96i1p32-34.

21. Panneflex LP, Salazar DA, Munive MV. Conocimientos, creencias y prácticas de los adolescentes de la cultura Caribe en anticoncepción. RevCuid. [Internet] 2016;7(1) [acesso em 12 ago 2017].Disponível: http://dx.doi.org/10.15649/ cuidarte.v7i1.243.

22. Duarte HHS, Bastos GAN, Duca GFD, Corleta HVE. Utilização de métodos contraceptivos por adolescentes do sexo feminino da Comunidade Restinga e Extremo Sul. Rev Paul Pediatr. [Internet] 2011;29(4) [acesso 02 fev 2018]. Disponível em: http://dx.doi.org/10.1590/S0103-05822011000400016. 